Med Klin Intensivmed Notfmed 2018 $113: 446-447$ https://doi.org/10.1007/s00063-018-0476-0 Online publiziert: 6 . August 2018

(c) Springer Medizin Verlag GmbH, ein Teil von Springer Nature 2018

CrossMark

\section{U. Janssens ${ }^{1} \cdot$ W. Druml ${ }^{2}$}

${ }^{1}$ Klinik für Innere Medizin und Internistische Intensivmedizin, Sankt Antonis-Hospital Eschweiler, Eschweiler, Deutschland

${ }^{2}$ Klinik für Innere Medizin III, Abteilung für Nephrologie, Allgemeines Krankenhaus Wien, Wien, Österreich

\title{
Organinteraktion in der Intensivmedizin
}

\section{Das Yin und Yang der Organe}

Unter physiologischen Bedingungen bestehen komplexe Interaktionen zwischen allen Organen zur Sicherstellung einer ausgewogenen Homöostase. Eine der Hauptaufgaben der Intensivmedizin besteht in der Überwachung und Überbrückung gestörter bzw. ausgefallener Organsysteme schwer erkrankter Patienten mit medikamentösen und/ oder interventionellen Verfahren. Dabei kommen regelhaft extrakorporale Organersatztherapien wie die maschinelle Beatmung, die Nierenersatztherapie oder extrakorporale Herz-Kreislauf-Unterstützungssysteme einzeln oder kombiniert zum Einsatz. Intensivmediziner wissen sehr wohl, dass es nicht ein singuläres Organversagen gibt, sondern dass man immer auf die Funktion, aber auch Dysfunktion der übrigen Organe achten und diese ebenfalls engmaschig überwachen muss. Das gilt insbesondere für Schockzustände jedweder Ursache. Hier besteht als zentrale Störung ein Missverhältnis zwischen Sauerstofftransport und Sauerstoffbedarf mit einer nachfolgenden kritischen Minderperfusion aller Organsysteme. Die schwere Sepsis bzw. der septische Schock ist ebenfalls an dieser Stelle exemplarisch zu nennen. Es handelt sich um eine Systemerkrankung, die über multiple Kaskaden im Rahmen der generalisierten Inflammation schwere Schäden im Bereich der Mikrozirkulation und auf zellulärer Ebene in nahezu allen Organen verursacht. Am Endpunkt dieser Prozesse steht regelhaft ein Multiorgandysfunktionssyndrom bzw. Multiorganversagen. Das Schwerpunktthema dieses Hefts richtet den Blick auf einzelne Organe und die Interaktionen mit dem gesamten Körper, aber auch auf einzelne physiologisch und pathophysiologisch eng miteinander gekoppelte Organe.

Der Beitrag von S.J. Klein et al. (https://doi.org/10.1007/s00063-0180472-4) beschäftigt sich mit der Interaktion von Lunge und Nieren bei kritisch kranken Patienten. Hier zeigen sich in besonderer Weise die relevanten bidirektionalen Interaktionen, die auf hämodynamische und proinflammatorische Effekte durch die mechanische Beatmung und/oder Volumenüberladung zurückzuführen sind, zum anderen auf vermehrt freigesetzte oder nicht mehr adäquat durch die Niere ausgeschiedene bzw. metabolisierte Mediatoren wie Interleukin-6 und Tumor-NekroseFaktor a. Die Autoren weisen darauf hin, dass es zum jetzigen Zeitpunkt keine Therapieansätze gibt, die kausal in die Achse zwischen Lunge und Niere eingreifen. Daher ist ein besonderes Augenmerk auf die Prävention durch eine lungenprotektive Beatmung und Vermeidung einer Volumenüberladung zu richten.

E. Egerer et al. (https://doi.org/10. 1007/s00063-018-0465-3) gehen auf die besonderen Interaktionen von Herz und Gehirn ein, die nicht nur in der Kunst und Literatur als Sinnbilder von Verstand und Gefühl des Menschen gelten. Sie benutzen dabei das Bild von „Täter- und Opferorganen“ und führen als Beispiel das Gehirn als „embolisches“ Opfer nach kardioembolisch bedingtem Insult bei Vorhofflimmern oder das
Herz mit einer schweren akuten TakoTsubo-Kardiomyopathie, bei der das Hirn dem Herzen schädlichen Stress bereitet, an. Sowohl die Herz-Hirn-Achse als auch die Hirn-Herz-Achse kann zu relevanten Erkrankungen des jeweilig anderen Organsystems beitragen. Dabei spielen die autonome Innervation des Herzens (Sympathikus und Parasympathikus), aber auch neuroendokrine Aspekte der Hypothalamus-Hypophysen-Nebennierenrinden-Achse neben der Inflammation eine zentrale Rolle.

Die Interaktionen von Leber und Lunge sind häufig, stehen aber nicht im Fokus bei der täglichen intensivmedizinischen Visite. V. Fuhrmann et al. (https://doi. org/10.1007/s00063-018-0473-3) weisen in ihrem Beitrag eindringlich darauf hin, dass Patienten mit akuten, akut-auf-chronischen und chronischen Lebererkrankungen häufig auch ein respiratorisches Organversagen aufweisen. Das hepatopulmonale Syndrom sowie die portopulmonale Hypertension finden sich häufig bei Patienten mit chronischer Lebererkrankung, insbesondere bei der Leberzirrhose. Folge sind pulmonalvaskuläre Veränderungen, parenchymatöse Veränderung der Lunge sowie extrapulmonale Veränderungen. Auch restriktive Ventilationsstörungen der Lunge infolge extrapulmonaler Krankheitsbilder (z. B. Aszites, hepatischer Hydrothorax) sind alltagsrelevant und sollten frühzeitig diagnostiziert und behandelt werden.

Der Darm als Motor des Multiorganversagens steht im Zentrum der Betrachtungen von W. Druml (https://doi.org/ 10.1007/s00063-018-0475-1). Der Darm 
ist ein entscheidendes immunologisches, metabolisches und neurologisches Organ, eine Störung seiner Funktionen korreliert mit Morbidität und Mortalität. Wichtigster Mechanismus der Entstehung endogener Infektionen ist die intestinale Translokation von Keimen. Die Erhöhung des intraabdominellen Drucks ist ein weiterer elementarer Mechanismus, der zu einer zusätzlichen Schädigung des Darms, zu einer $\mathrm{Zu}$ nahme der Translokation sowie Inflammation und Beeinträchtigung anderer Organsysteme wie Niere, Kreislauf und Lunge beiträgt.

Liebe Leserinnen und Leser, die Beschäftigung mit den einzelnen Organen, den Interaktionen zwischen den Organen und der damit zusammenhängenden Pathophysiologie ist sicherlich sehr komplex, aber unverzichtbar für die tägliche klinische Arbeit und auch für eine erfolgreiche Intensivtherapie. Wir möchten den Autorinnen und Autoren für ihre exzellenten Beiträge und für die damit verbundene Mühe und den Einsatz von Zeit danken.

Wir wünschen Ihnen eine spannende und aufschlussreiche Lektüre!

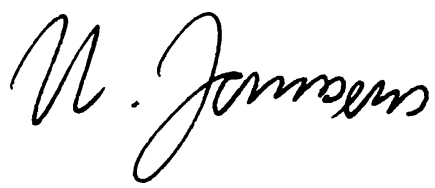

Prof. Dr. med. Uwe Janssens

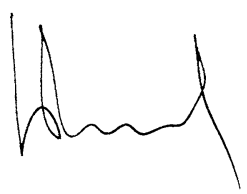

Prof. Dr. med. Wilfred Druml

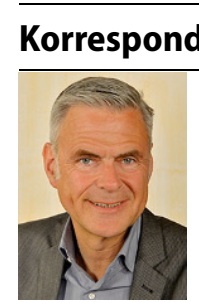

DRKS: 10 Jahre Transparenz bei klinischen Studien

Informationen zu rund 7200 Studien

Prof. Dr. U. Janssens

Klinik für Innere Medizin und Internistische

Intensivmedizin, Sankt

Antonis-Hospital Eschweiler

Dechant-Deckers-

Straße 8, 52249 Eschweiler,

Deutschland

uwe.janssens@

sah-eschweiler.de

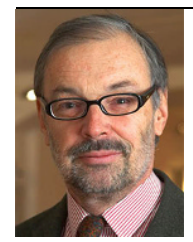

Prof. Dr. W. Druml

Klinik für Innere Medizin III, Abteilung für Nephrologie, Allgemeines Krankenhaus Wien

Währinger Gürtel 18-20, 1090 Wien, Österreich wilfred.druml@ meduniwien.ac.at

Interessenkonflikt. U. Janssens und W. Druml geben an, dass kein Interessenkonflikt besteht.

Das Deutsche Register Klinischer Studien (DRKS) verweist anläßlich seines 10-jährigen Jubiläums darauf, dass inzwischen auf Informationen zu rund $\mathbf{7 2 0 0}$ hierzulande durchgeführten klinischen Studien zugegriffen werden kann.

Das Deutsche Register Klinischer Studien (DRKS) bietet die Möglichkeit, Informationen zu laufenden und abgeschlossenen klinischen Studien in Deutschland zu suchen oder eigene Studien über die Registrierung anderen zugänglich zu machen. Zu jeder Studie finden sich Eckdaten wie Studientitel, Kurzbeschreibungen, Ein- und Ausschlusskriterien, Studienstatus und Endpunkte. Das Register hilft unter anderem, Teilnehmer für Studien zu finden oder Doppelstudien zu vermeiden. Vor allem aber bietet es einfach zugängliche Informationen und unterstützt so Ethikkommissionen und Behörden, die klinische Studien begutachten müssen, so das DRKS.

\section{WHO-Primärregister}

Das International Committee of Medical Journal Editors (ICMJE) fordert, Beiträge zu Studien nur zu veröffentlichen, wenn diese rechtzeitig in einem anerkannten Register registriert wurden. Dem haben sich zahlreiche medizinische Journals angeschlossen. Mit einer Registrierung im DRKS als WHO-Primärregister sind die Anforderungen des ICMJE als Voraussetzung für eine Veröffentlichung erfüllt.

\section{Beim DIMDI angesiedelt}

Das DRKS ist kostenfrei und öffentlich zugänglich und wird vom Deutschen Institut für Medizinische Dokumentation und Information (DIMDI) als Behörde im Ressort des Bundesministeriums für Gesundheit (BMG) betrieben.

Quelle: Deutsches Register Klinischer Studien (DRKS) www.drks.de 